\title{
Jóvenes y fake news. Un análisis sociodemográfico aplicado al caso andaluz
}

Fake news and youth. A socio-demographic analysis applied to the andalusian case

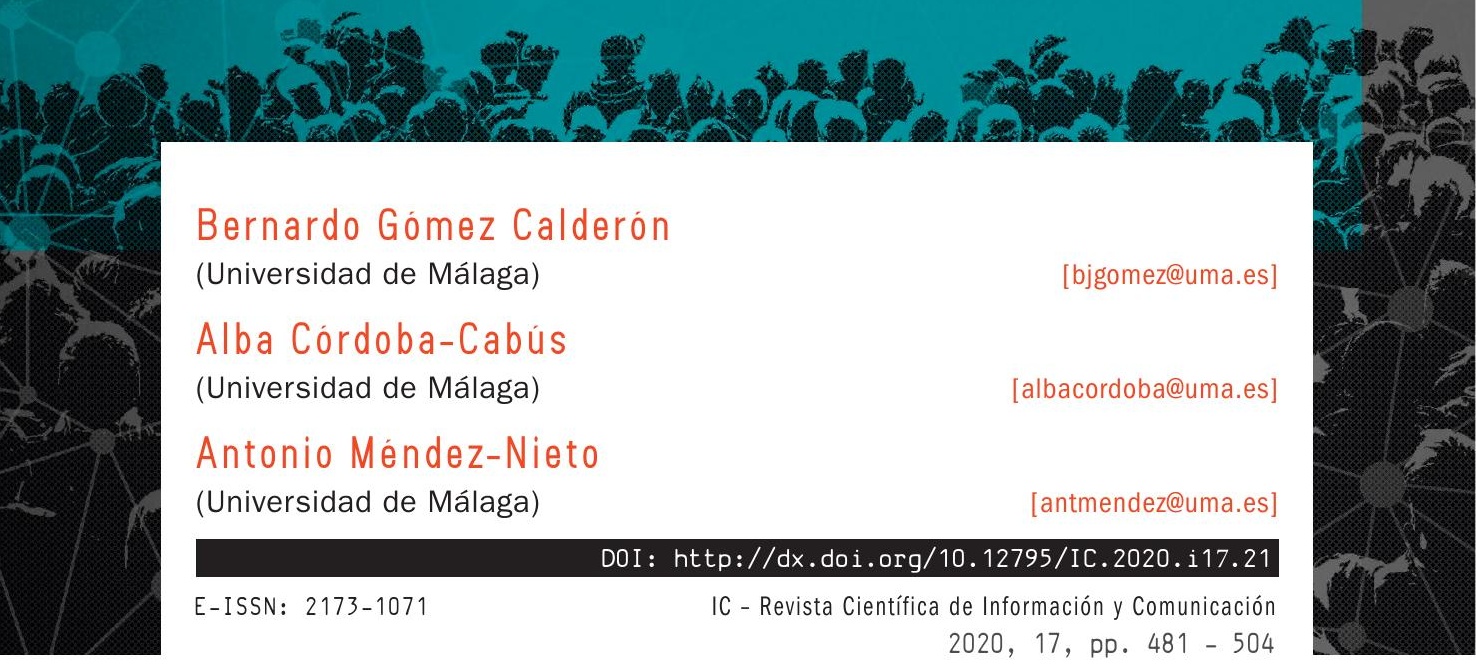

\section{Resumen}

En este estudio se aborda la incidencia de las noticias falsas entre los jóvenes andaluces, a partir de un cuestionario realizado en las ocho provincias de la comunidad a individuos de entre 15 y 24 años $(n=1.068)$. Los resultados revelan que el colectivo juvenil se encuentra intensamente expuesto a las fake news, con diferencias a veces significativas en función de las características sociodemográficas de los encuestados.

\section{Abstract}

In this paper we analyse the incidence of fake news among Andalusian young population with a survey carried out to individuals between 15 and 24 from the eight provinces of the region $(n=1.068)$. The results reveal that Andalusian youth is widely exposed to fake news, and show significant differences according to the socio-demographic characteristics of the respondents.

\section{Palabras clave}

Fake news, redes sociales, jóvenes, España, Andalucía 


\section{Keywords}

Fake news, social media, youth, Spain, Andalusia

\section{Sumario}

1. Introducción: el "hybrid media system" y las redes sociales como canal informativo

1.1. La eclosión de las fake news

1.2. Los jóvenes españoles ante las fake news. El caso andaluz

2. Metodología

3. Resultados

3.1. Frecuencia de recepción de fake news

3.2. Temas de las fake news

3.3. Fuentes originarias de las fake news

3.4. Verificación de las fake news

3.5. Fuentes empleadas para verificar las fake news

4. Conclusiones

5. Bibliografía

\section{Summary}

1. Introduction: "hybrid media system" and social media as informative channel

1.1. The hatching of fake news

1.2. Fake news and the Spanish youth. The Andalusian case

2. Method

3. Results

3.1. Fake news reception

3.2. Fake news topics

3.3. Fake news sources

3.4. Fake news checking

3.5. Sources for fake news checking

4. Conclusions

5. Bibliography 


\section{Introducción: el "hybrid media system" y las redes sociales como canal informativo}

La implantación de la sociedad de la información, materializada en la digitalización de los contenidos y en su difusión inmediata por medio de terminales electrónicos, ha implicado cambios profundos en el sector periodístico (García Santamaría, 2014), en el modo en que se componen y difunden las noticias y en cómo se financian. Además, ha traído aparejada la multiplicación de los emisores, pues a los medios convencionales, con sus ingentes estructuras de producción, se ha sumado un conjunto de entidades heterogéneas a través de las cuales las noticias se difunden con igual eficacia -y a veces más- que mediante los soportes clásicos, dando lugar a un auténtico "hybrid media system" (Chadwick, 2013). Esas entidades serían los portales agregadores de noticias, los blogs personales y, muy especialmente, las redes sociales (Guallar et al., 2016), ágoras contemporáneas en las cuales cada usuario es un emisor en potencia y la información se expande de modo descentralizado.

A la hora de proporcionar noticias nacionales e internacionales, los social media parecen sustituir, entre capas cada vez más amplias de la población, a los medios de comunicación tradicionales, y lo hacen prácticamente en todo el mundo (Newman, Dutton \& Blank, 2012; Nielsen \& Schrøder, 2014; Bakshy, Messing \& Adamic, 2015; Gottfried \& Shearer, 2017).

En el caso de España, el Digital News Report.es recoge en su última edición (2019) que el $17 \%$ de la población prefiere las redes sociales y los blogs para acceder a la información de actualidad, en detrimento de los medios, oscilando por tramos de edad entre el público de 18 a 24 años, donde la proporción se eleva hasta el 38\%, y el mayor de 65 , que solo se decanta por las social networks en un $5 \%$ de los casos.

Por lo que respecta a Andalucía, el Consejo Audiovisual de la comunidad registra en su último barómetro anual (2019) que el 38,2\% de la audiencia se informa a través de las redes, siendo la población activa -tanto empleados como paradosy los estudiantes el público preferente de las nuevas plataformas. La tasa se ha incrementado de modo considerable desde el ejercicio 2011, cuando se situaba en un $19,9 \%$. 


\subsection{La eclosión de las fake news}

Es en este contexto informativo híbrido y descentralizado en el que cobra impulso el fenómeno de las fake news ${ }^{1}$. Su concurso - parece que notable- en dos procesos democráticos celebrados en 2016, la consulta británica sobre el Brexit y las elecciones presidenciales estadounidenses que ganó Donald Trump, atrajo sobre ellas el foco de los medios de comunicación y los gobiernos de todo el mundo. De acuerdo con la estadística llevada a cabo por empresas de medición de tráfico de datos, cientos de bulos circularon ampliamente a través de las redes sociales, en defensa tanto del Brexit (Bastos \& Mercea, 2017) como de la candidatura de Trump (Allcott \& Gentzkow, 2017; Bakir \& McStay, 2018; Bovet \& Makse, 2019; Magallón, 2019a), durante las semanas que duraron sus respectivas campañas. En el caso británico, las informaciones destacaban los supuestos agravios que el Reino Unido sufría en su condición de socio comunitario, y las ventajas -o mejor dicho, la ausencia de costes directos- que tendría para el país la salida de la UE (Grice, 2017). En cuanto a las presidenciales norteamericanas, la mayor parte de las noticias falsas se dirigían contra Hillary Clinton, que fue acusada, entre otras cosas, de vender armas al Dáesh y de formar parte de una red de prostitución infantil (Silverman, 2016).

Desde entonces, han sido numerosos los acontecimientos de índole políticosocial en los que las fake news se han visto involucradas: el referéndum por la paz en Colombia, celebrado en octubre de 2016 (Fernández-García, 2017; Pauner Chulvi, 2018); la consulta catalana del 1-0 de 2017 (Alandete, 2019), el caso Fillon en Francia (Baider \& Constantinou, 2017), las presidenciales brasileñas de 2018 ganadas por Jair Bolsonaro (Oliveira \& Rossi, 2018), las dos elecciones generales celebradas en España en 2019 (Magallón, 2019b) y, aún en pleno desarrollo, la crisis mundial derivada de la pandemia del coronavirus SARS-Cov-2 (Brennen et al., 2020; Masip et al., 2020), por citar solo algunos casos.

Al hilo de los acontecimientos de actualidad, la academia ha comenzado a ocuparse del fenómeno de las fake news, con dos objetivos prioritarios: desvelar los mecanismos que las convierten en artefactos tan eficaces, y proveer a la audiencia de un repertorio de estrategias defensivas que minimicen o anulen el efecto de estos contenidos malintencionados.

1 Se emplea esta denominación aquí, en lugar de su equivalente false news, por ser el modo más popular de referirse al fenómeno, pese a que algunos autores han denunciado ya el carácter tendencioso del término debido a su uso partidista en los Estados Unidos, donde cualquier información crítica con el gobierno de Trump es calificada por las autoridades como fake (cf. Vosoughi, Roy y Aral, 2018). 
Es obvio que las noticias falsas han existido siempre, desde la aparición de los canales de difusión masiva de información, y que constituyen uno de los pilares de la propaganda (Taylor, 2013; Palmer, 2017; Parra \& Oliveira, 2018). En nuestros días, simplemente, presentan la particularidad de haber encontrado una vía para extenderse a enorme velocidad entre millones de individuos, a través de las redes sociales. Aunque se trata de una denominación de límites un tanto imprecisos (cf. Gelfert, 2018), en sentido estricto hemos de entender las fake news como "news articles that are intentionally and verifiably false, and could mislead readers" (Allcot \& Gentzkow, 2017, p. 213), de aspecto similar a los textos periodísticos convencionales (Tandoc, Jenkins \& Craft, 2019). Excluimos de esta categoría los contenidos de actualidad que desinforman de modo involuntario debido a que los reporteros creen correctos los datos que suministran.

Junto con las redes sociales, las páginas web constituyen el principal foco generador de noticias falsas; suelen ser sitios de apariencia neutral y propósito supuestamente informativo en los cuales se fabrican la mayor parte de los bulos que pronto se convierten en virales. En algunos casos, los promotores de dichas iniciativas no dejan de resultar pintorescos, como los más de cien adolescentes de un municipio de Macedonia que generaron decenas de historias ficticias en torno a las elecciones presidenciales estadounidenses de 2016 (Subramanian, 2017).

Los primeros análisis Iongitudinales parecen confirmar el enorme poder expansivo de las fake news. De acuerdo con Vosoughi, Roy y Aral (2018), los bulos se difunden a través de las redes sociales de forma más extensa, rápida y profunda que las informaciones veraces, sea cual sea su temática pero sobre todo si están relacionados con la actualidad política. El análisis pormenorizado de 126.000 afirmaciones fraudulentas difundidas por Twitter entre 2006 y 2017 permitió a estos autores constatar que, de media, las noticias falsas reciben un $70 \%$ más de retuits que las verdaderas. Su hipótesis es que triunfan tan ampliamente porque suelen provocar entre la audiencia respuestas de indignación, temor y sorpresa; y concluyen que, en su difusión, los conocidos como bots, cuentas teledirigidas que actúan como repetidores automáticos de mensajes, desempeñan un papel mucho más discreto del que algunos estudios (cf. Shao et al., 2017) les han atribuido: "Human behavior contributes more to the differential spread of falsity and truth than automated robots do", concluyen (2018: 1.150).

Según Allcot y Gentzkow (2017: 217), dos parecen ser los motivos principales que fomentan la producción de fake news: uno, obvio, el económico, ya que los artículos que se viralizan en las redes proporcionan considerables beneficios publicitarios cada vez que consiguen que los usuarios, a través de enlaces, visiten las páginas matrices. El 
otro motor sería ideológico: desacreditar a políticos cuyas posturas no se comparten. A estos dos factores, nosotros sumaríamos un tercero, el estratégico, cuando los bulos los facturan instituciones, o incluso países, que desean fortalecer su posición o minar la de sus oponentes; sería el caso, por ejemplo, de las noticias falsas supuestamente generadas desde Rusia en apoyo de la candidatura presidencial de Donald Trump (Magallón, 2019a).

Las taxonomías que tratan de acotar el fenómeno fijan para él márgenes diversos. Allcott y Gentzkow (2017) distinguen seis tipos de fake news: informaciones con errores no intencionados, rumores, teorías de la conspiración, sátiras mal interpretadas, falsas declaraciones de políticos e informes distorsionados o tendenciosos. Por su parte, Tandoc, Wei Lim y Ling (2018) proponen una clasificación más amplia, que estaría compuesta por noticias satíricas, parodias de noticias, noticias fabricadas, noticias manipuladas, publicidad -o más bien infopublicidad-y propaganda.

La capacidad de la audiencia para identificar un contenido informativo falso es difícil de determinar, ya que depende en gran medida de la autopercepción de los individuos, un valor muy poco objetivo. De ahí que sea habitual toparse aquí con registros discordantes: por citar solo dos casos, mientras que el ler estudio sobre el impacto de las fake news en España estima que el $86 \%$ de la población tiene dificultades para reconocer un bulo (Amorós, 2018), la tasa de habitantes de la UE que se considera preparada para detectar noticias falsas asciende al 52\%, según el último Eurobarómetro (Comisión Europea, 2019).

Sobre el efecto real que las fake news tienen en el comportamiento de la ciudadanía los investigadores se muestran mayoritariamente escépticos, al menos en términos electorales, que son los concurrentes en los casos del Brexit y las victorias de Trump y Bolsonaro (Allcot \& Gentzkow, 2017). No obstante, en otras esferas, sus consecuencias pueden resultar dramáticas, como en el atentado de la maratón de Boston (Salas, 2018) o en el denominado "Pizzagate”, que casi acaba en masacre (Griffin, 2016). Quizá por ello la audiencia tiende a atribuir a las informaciones falsas un notable poder de influencia (Carrión, 2017). Del mismo modo, también puede resultar relevante, según algunos autores, el impacto de las fake news a la hora de fijar "temas, tendencias y posiciones en el debate público" (Hernández-Conde \& Fernández García, 2019: 36).

\subsection{Los jóvenes españoles ante las fake news. El caso andaluz}

En todos los países sometidos a estudio hasta la fecha -en su mayoría, desarrollados-, el acceso a contenidos noticiosos a través de las redes sociales se da 
de forma especialmente habitual entre los usuarios más jóvenes, que utilizan los social media con mayor frecuencia e intensidad que el resto de la población y desconocen modos alternativos de acceder a las noticias, ya que cada vez frecuentan menos los medios de comunicación tradicionales (García, Gértrudix \& Gértrudrix, 2014; Mitchelstein \& Boczkowski, 2018; Center for Internet Studies and Digital Life, 2019).

Aunque algunos autores consideran que conviene desconfiar de la capacidad de los jóvenes para detectar bulos, pese a ser nativos digitales (Wineburg \& McGrew, 2017), parece un segmento de población razonablemente escéptico ante la información masiva (Madden, Lenhart \& Fontaine, 2018) y al que le preocupa el auge de las fake news (Antunes \& Nina, 2020). De hecho, según Catalina-García, Vozmediano y García-Jiménez (2017), en el caso de España hay un alto porcentaje de jóvenes que suele contrastar las noticias que recibe a través de los social media.

A escala nacional, se han efectuado ya algunas aproximaciones a la percepción que tiene el colectivo juvenil sobre las fake news, aunque con muestras algo sesgadas en la medida en que suelen estar constituidas exclusivamente por alumnos universitarios, que pueden considerarse a priori más preparados para detectar y desestimar bulos. De acuerdo con Catalina-García, Sousa y Cristina-Silva (2019), el 92,7\% del segmento usa redes sociales con fines informativos, de manera preferente el servicio de mensajería instantánea WhatsApp (40\%). De ellos, el 16\% asegura verificar las noticias que recibe "siempre", y un $83 \%$, a veces. La percepción sobre la propia capacidad para identificar contenidos fraudulentos se cifra en 6,8 sobre 10 , aunque el hecho de que la muestra la integren solo estudiantes de Ciencias de la Comunicación - por defecto, mejor adiestrados- puede implicar cierto sesgo. Las temáticas más frecuentes de este tipo de mensajes son la política, el deporte (39\% en ambos casos) y la economía (25\%).

En un estudio circunscrito solo a estudiantes universitarios del País Vasco, Mendiguren, Pérez y Meso (2020) obtienen proporciones algo más elevadas: el 80\% de los encuestados cree que tiene criterio para identificar las fake news, que le llegan principalmente a través de las redes sociales, en especial Facebook, Twitter y WhatsApp. La política, los temas sociales y la economía serían los asuntos más habituales de las noticias que reciben, y en cuanto a las fuentes empleadas para contrastarlas, se apunta a medios tradicionales, diarios digitales y al motor de búsqueda Google.

En el caso de Andalucía, ámbito geográfico de nuestro estudio, disponemos ya de algunas constataciones. Así, según Herrero-Diz et al. (2019), los jóvenes con formación en periodismo y comunicación tendrían más facilidad para detectar informaciones falsas que el resto. También sostienen estos autores que los contenidos de sátira política que 
imitan el formato noticioso generan una confusión notable entre la audiencia, e incluso apuntan a un posible sesgo de género en este terreno -las mujeres confiarían más en textos de intención paródica-, aunque con cautelas.

\section{Metodología}

A la vista de los antecedentes descritos, esta investigación se planteó con el propósito básico de determinar la incidencia del fenómeno de las fake news entre los jóvenes andaluces de entre 15 y 24 años. Como objetivos subsidiarios del estudio, los autores se propusieron conocer la frecuencia con que los usuarios reciben noticias falsas a través de redes sociales y servicios de mensajería instantánea (01), establecer las características predominantes de dichas noticias así como sus fuentes de procedencia más habituales (02) y, finalmente, explorar la reacción de los jóvenes ante los contenidos que consideran sospechosos, en qué medida cotejan los datos con fuentes alternativas y cuál es la naturaleza de las entidades a las que recurren para verificar la información (03).

Para alcanzar los objetivos de la investigación, se optó por aplicar la técnica del cuestionario, tomando como universo la población de entre 15 y 24 años residente en Andalucía, fijada en 891.218 personas en el momento de la realización del estudio (INE, 2018).

Se partió de un muestreo proporcional a los valores teóricos del universo, pero al no ser posible alcanzar cuotas satisfactorias por sexo, edad y provincia, se aplicaron los coeficientes de ponderación estimados. Para un nivel de confianza del 95\%, la muestra se estableció en 1.066 encuestas (se alcanzaron 1.068, un 100,1\% del total) y el margen de error obtenido fue de $+/-3 \%$.

TABLA 1

Composición de la muestra

\begin{tabular}{c|c|c|c|c|c|}
\hline & \multicolumn{3}{c|}{ Hombres } & \multicolumn{3}{c|}{ Mujeres } \\
\cline { 2 - 5 } & \multicolumn{1}{c|}{$15-20$ años (\%) } & $21-24$ años (\%) & $15-20$ años (\%) & $21-24$ años (\%) & Total (\%) \\
\hline Almería & 0,3 & 1,2 & 0,8 & 2,2 & 4,5 \\
\hline Cádiz & 1,6 & 4,3 & 1,2 & 5,2 & 12,4 \\
\hline
\end{tabular}




\begin{tabular}{c|c|c|c|c|c|}
\hline Córdoba & 0,9 & 1,1 & 2,1 & 4,4 & 8,5 \\
\hline Granada & 1,6 & 4,1 & 2,5 & 6,7 & 15,0 \\
\hline Huelva & 0,3 & 1,1 & 0,7 & 2,7 & 4,9 \\
\hline Jaén & 0,8 & 1,6 & 1,0 & 3,6 & 7,0 \\
\hline Málaga & 1,3 & 5,1 & 2,6 & 8,3 & 17,4 \\
\hline Sevilla & 2,1 & 10,5 & 4,9 & 12,9 & 30,3 \\
\hline Total & 8,9 & 29,1 & 15,9 & 46,1 & 100,0 \\
\hline
\end{tabular}

El cuestionario se componía de diez preguntas, de respuesta única o múltiple según los casos, y fue suministrado vía online a los encuestados entre el 30 de mayo y el 4 de junio de 2019. Con los registros obtenidos se generó una matriz de datos en SPSS que, tras la realización de análisis descriptivos e inferenciales, permitió comprobar las relaciones entre las variables dependientes e independientes incluidas en el estudio ${ }^{2}$. Primero, se calculó el estadístico de contraste chi-cuadrado $\left(\mathrm{X}^{2}\right)$, con la corrección de continuidad de Yates $\left(\mathrm{X}_{\mathrm{y}}^{2}\right)$ en caso de tener un grado de libertad, para decidir si existía dependencia. Posteriormente, se analizaron los residuos tipificados corregidos para indicar la dirección de la relación. Por último, se evaluó el tamaño del efecto (TE), teniendo en cuenta el nivel de medida de las variables para la selección del estadístico adecuado: coeficiente de Phi, coeficiente de contingencia, V de Cramer o Eta.

\section{Resultados}

A continuación, se detallan los hallazgos obtenidos, haciendo hincapié en las asociaciones encontradas entre las variables de estudio.

2 Las variables independientes eran seis: edad, sexo, provincia, hábitat, nivel de estudios y ocupación de los entrevistados. 


\subsection{Frecuencia de recepción de fake news}

Los datos muestran que los jóvenes andaluces de entre 15 y 24 años reciben con suma frecuencia noticias falsas a través de redes sociales y aplicaciones de comunicación instantánea. Únicamente el 5,9\% de los encuestados asegura no haber recibido nunca ese tipo de contenidos, mientras que el resto $(94,1 \%)$ confirma que le llegan al menos una vez al mes. Como puede apreciarse en la distribución (tabla 2), el grueso de las incidencias oscila entre alguna vez a la semana $(24,9 \%)$, varias veces a la semana $(23,3 \%)$ y alguna vez al mes $(21,6 \%)$.

\section{TABLA 2}

\section{Frecuencia con que se reciben fake news}

\begin{tabular}{c|c|c}
\hline & Casos & $\%$ \\
\hline Varias veces al dia & 80 & 7,5 \\
\hline Todos los dias & 178 & 16,8 \\
\hline Varias veces a la semana & 247 & 23,3 \\
\hline Alguna vez a la semana & 264 & 24,9 \\
\hline Alguna vez al mes & 228 & 21,6 \\
\hline Nunca & 62 & 5,9 \\
\hline Total & 1.059 & 100,0 \\
\hline
\end{tabular}

De acuerdo con la prueba de independencia chi-cuadrado, $\left[\chi^{2}(30, N=1.058)=\right.$ $46,228, p<0,05]$, existe asociación entre la frecuencia con que se reciben noticias falsas y el nivel formativo de los encuestados ( $T E=0,209)$. En este punto, se podría decir que la audiencia parece estar más expuesta a contenidos informativos fraudulentos cuanto mayor es su nivel de estudios.

\subsection{Temas de las fake news}

Las noticias falsas a las que de modo más habitual accede la juventud andaluza están relacionadas sobre todo con la actualidad social, en concreto, con la actividad de 
personajes de la vida pública (64,3\% de respuestas). A estas les siguen las informaciones referidas a política $(62,8 \%)$, economía (37\%), cultura, deportes (ambas categorías con un $19,1 \%$ de incidencias) y otros $(4,1 \%)^{3}$. En esta última modalidad destacan, por encima del resto, los contenidos vinculados a acontecimientos luctuosos o de carácter delictivo, como desapariciones, violaciones o estafas.

Los resultados del estadístico de contraste chi-cuadrado arrojan asociaciones significativas entre la temática de las fake news y el sexo y el nivel formativo de los encuestados. En este sentido, como se plasma en la tabla 3, el género influye en la recepción de noticias sobre sociedad ( $T E=0,155)$, política $(T E=0,173)$, cultura $(T E=0,076)$ y deportes $(\mathrm{TE}=0,146)$, mientras que en las que abordan otros asuntos no existen diferencias entre grupos. El análisis de residuos tipificados corregidos indica que la recepción de informaciones falsas sobre política y deportes por parte de los hombres, y la de sociedad y cultura por parte de las mujeres, divergen de forma significativa.

TABLA 3

Temas de las fake news recibidas en función del sexo de los encuestados*

\begin{tabular}{|c|c|c|c|c|c|c|c|c|}
\hline & \multicolumn{2}{|c|}{ Sociedad } & \multicolumn{2}{|c|}{ Politica } & \multicolumn{2}{|c|}{ Cultura } & \multicolumn{2}{|c|}{ Deportes } \\
\hline & No $(\%)$ & Si $(\%)$ & № $(\%)$ & Si $(\%)$ & № (\%) & Si $(\%)$ & No $(\%)$ & Si (\%) \\
\hline Hombre & 42,8 & 57,2 & 29,2 & 70,8 & 83,7 & 16,3 & 75,4 & 24,6 \\
\hline \multirow[t]{2}{*}{ Mujer } & 27,9 & 72,1 & 45,9 & 54,1 & 77,7 & 22,3 & 86,9 & 13,1 \\
\hline & \multicolumn{2}{|c|}{$\begin{array}{l}\chi_{y}^{2}(1, N=997)= \\
23,287, p<0,05\end{array}$} & \multicolumn{2}{|c|}{$\begin{array}{l}\chi_{y}^{2}(1, N=996)= \\
29,038, p<0,05\end{array}$} & \multicolumn{2}{|c|}{$\begin{array}{c}\chi_{y}^{2}(1, N=997)= \\
5,316, p<0,05\end{array}$} & \multicolumn{2}{|c|}{$\begin{array}{l}\chi_{y}^{2}(1, N=996)= \\
20,604, p<0,05\end{array}$} \\
\hline
\end{tabular}

En cuanto a la formación de los entrevistados, se observa que esta condiciona la recepción-o mejor dicho, la identificación- de fake news sobre sociedad $(\mathrm{TE}=0,141)$ y cultura $(T E=0,137)$, sin que se observe dependencia en el resto de las temáticas. Como se aprecia en la tabla 4, la detección de noticias falsas sobre asuntos culturales se incrementa

3 El cuestionario permitía marcar en esta pregunta tantas opciones como fueran necesarias. 
levemente a medida que aumenta el nivel de estudios, y algo similar ocurre, aunque de forma más discreta, con la variable sociedad.

TABLA 4

Temas de las fake news recibidas en función del nivel formativo de los encuestados ${ }^{*}$

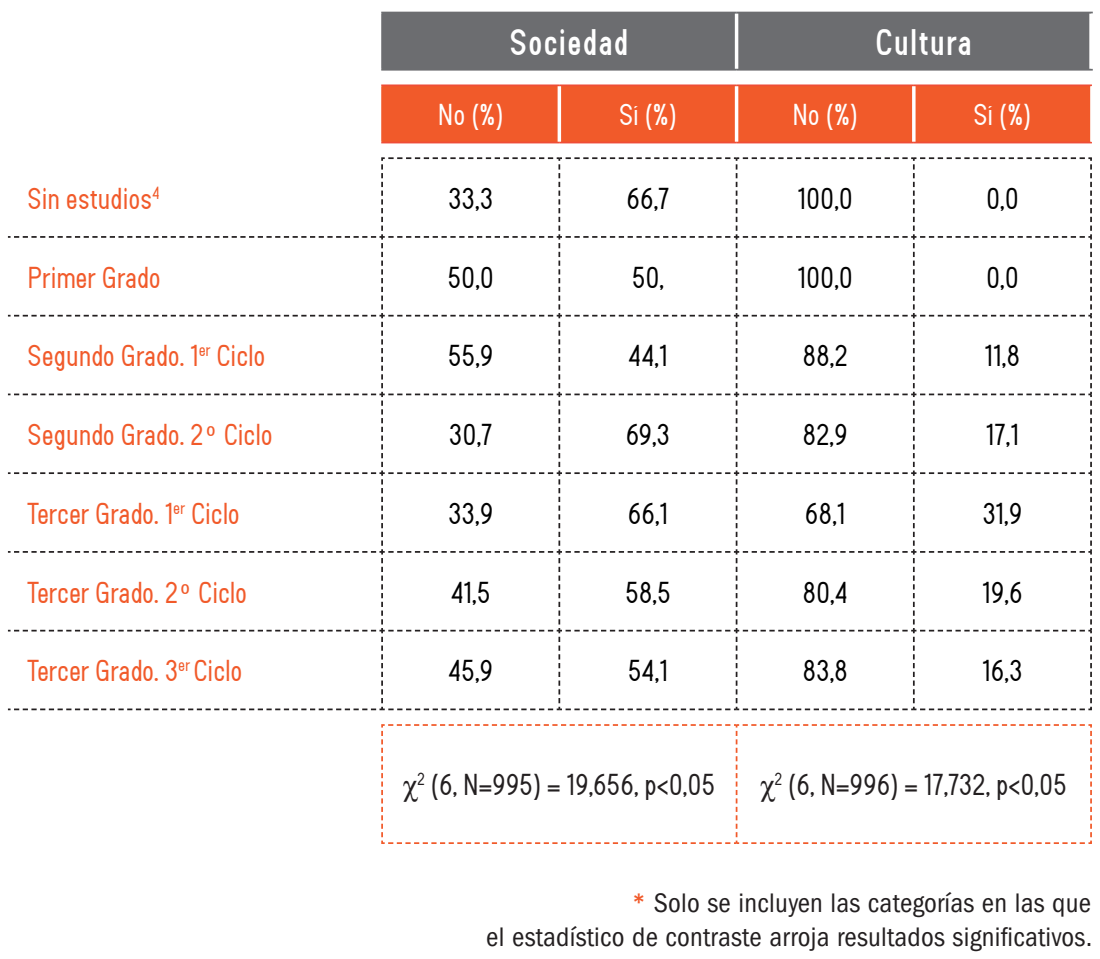

4 La correspondencia de los niveles educativos es la siguiente: Sin estudios = no ha finalizado la educación primaria; Primer Grado = primera etapa de EGB o educación primaria completada; Segundo Grado $1^{\text {er }}$ Ciclo = graduado escolar $01^{\circ}$ y $2^{\circ}$ de ESO; Segundo Grado $2^{\circ}$ Ciclo = BUP, COU, ESO completada, FP grado medio o Bachillerato; Tercer Grado $1^{\text {er }}$ Ciclo $=$ diplomatura, ingeniería técnica, título de escuela superior o FP grado superior; Tercer Grado $2^{\circ} \mathrm{Ciclo}=$ licenciatura, grado 0 ingeniería superiore; Tercer Grado $3^{\text {er }}$ Ciclo = máster 0 doctorado. 


\subsection{Fuentes originarias de las fake news}

Los jóvenes andaluces de entre 15 y 24 años reciben noticias falsas principalmente de tres fuentes: medios de comunicación generalistas como diarios, televisiones o radios $(50,4 \%)$; líderes políticos o partidos $(45,2 \%)$ y youtubers o influencers a los que siguen $(41,6 \%)^{5}$. En menor medida, las informaciones emanan de familiares o amigos $(38,4 \%)$; empresas de cualquier sector, exceptuando las de medios $(37,7 \%)$; webs especializadas $(18,9 \%)$ y organizaciones y asociaciones $(18,5 \%)$.

La prueba de chi-cuadrado revela que, en algunas ocasiones, existe asociación significativa entre el tipo de fuente que emite la noticia falsa y el nivel formativo de los jóvenes. Como se muestra en la tabla 5, esta influencia se presenta sobre todo cuando las entidades son empresas $(T E=0,168)$, organizaciones y asociaciones $(T E=0,127)$, medios de comunicación generalistas ( $\mathrm{TE}=0,126)$ y youtubers o influencers $(\mathrm{TE}=0,115)$.

\section{TABLA 5}

\section{Fuente originaria de las noticias falsas en relación con la formación} del encuestado*

\begin{tabular}{|c|c|c|c|c|c|c|c|c|}
\hline & \multicolumn{2}{|c|}{$\begin{array}{l}\text { Medios de } \\
\text { comunicación }\end{array}$} & \multicolumn{2}{|c|}{$\begin{array}{l}\text { Youtubers/ } \\
\text { influencers }\end{array}$} & \multicolumn{2}{|c|}{ Empresas } & \multicolumn{2}{|c|}{$\begin{array}{l}\text { Organizaciones } \\
\text { y asociaciones }\end{array}$} \\
\hline & No $(\%)$ & Si $(\%)$ & № $(\%)$ & Si $(\%)$ & № (\%) & Si $(\%)$ & No $(\%)$ & Si (\%) \\
\hline Sin estudios & 50,0 & 50,0 & 80,0 & 20,0 & 100,0 & 0,0 & 100,0 & 0,0 \\
\hline Primer Grado & 71,4 & 28,6 & 42,9 & 57,1 & 100,0 & 0,0 & 33,3 & 66,7 \\
\hline Segundo Grado. $1^{\text {er }}$ Ciclo & 60,5 & 39,5 & 71,1 & 28,9 & 55,3 & 44,7 & 89,5 & 10,5 \\
\hline Segundo Grado. $2^{\circ}$ Ciclo & 53,0 & 47,0 & 54,5 & 45,5 & 66,5 & 33,5 & 80,5 & 19,5 \\
\hline Tercer Grado. $1^{\text {er }}$ Ciclo & 35,8 & 64,2 & 69,9 & 30,1 & 53,7 & 46,3 & 86,2 & 13,8 \\
\hline Tercer Grado. $2^{\circ}$ Ciclo & 45,5 & 54,5 & 59,5 & 40,5 & 52,4 & 47,6 & 83,6 & 16,4 \\
\hline \multirow[t]{2}{*}{ Tercer Grado. $3^{\text {er Ciclo }}$} & 51,1 & 48,9 & 59,6 & 40,4 & 72,0 & 28,0 & 75,5 & 24,5 \\
\hline & \multicolumn{2}{|c|}{$\begin{array}{c}\chi^{2}(6, N=1059)= \\
16,870, p<0,05\end{array}$} & \multicolumn{2}{|c|}{$\begin{array}{c}\chi^{2}(6, N=1059)= \\
14,618, p<0,05\end{array}$} & \multicolumn{2}{|c|}{$\begin{array}{c}\chi^{2}(6, N=1057)= \\
29,730, p<0,05\end{array}$} & \multicolumn{2}{|c|}{$\begin{array}{c}\chi^{2}(6, N=1057)= \\
17,073, p<0,05\end{array}$} \\
\hline
\end{tabular}

* Solo se incluyen las categorías en las que el estadístico de contraste arroja resultados significativos.

5 En este caso, los encuestados podían seleccionar hasta tres opciones. 
En lo referente a los medios de comunicación, el grupo que parece recibir más fake news de esta fuente lo conforman individuos que disponen de un título superior de primer ciclo $(64,2 \%)$, aunque también son altos los porcentajes alcanzados aquí por jóvenes con licenciatura o grado (54,5\%) y máster o doctorado (48,9\%).

Frente a ello, se obtiene que los encuestados con menor nivel educativo son los más expuestos a informaciones falsas emanadas de youtubers o influencers $(57,1 \%$ de casos), en el otro extremo del arco profesional.

\subsection{Verificación de las fake news}

La práctica totalidad de la juventud andaluza de entre 15 y 24 años asegura recurrir a fuentes alternativas para contrastar las noticias cuando sospecha que son falsas (90,3\% de respuestas afirmativas). En el $36 \%$ de los casos las corroboran "habitualmente", "siempre" en el $30 \%$ y "a veces" en el $24,3 \%$. Parecen ser por tanto poco numerosos $(9,7 \%)$ quienes no practican algún tipo de verificación.

En esta variable interviene de modo significativo tanto la formación de los encuestados ( $T E=0,249$ ) como el hábitat en el que residen ( $T E=0,139)$. Así, cuanto mayor es el nivel de estudios, más frecuente resulta la comprobación de noticias de veracidad dudosa (tabla 6).

TABLA 6

Frecuencia de contraste de la información según nivel formativo de los encuestados

\begin{tabular}{|c|c|c|c|c|c|}
\hline & $\begin{array}{c}\text { Siempre } \\
(\%)\end{array}$ & $\begin{array}{c}\text { Habitualm. } \\
(\%)\end{array}$ & $\begin{array}{c}\text { A veces } \\
(\%)\end{array}$ & $\begin{array}{c}\text { Rara vez } \\
(\%)\end{array}$ & $\begin{array}{c}\text { Nunca } \\
(\%)\end{array}$ \\
\hline Sin estudios & 0,0 & 0,0 & 50,0 & 16,7 & 33,3 \\
\hline Primer Grado & 16,7 & 50,0 & 16,7 & 16,7 & 0,0 \\
\hline Segundo Grado. $1^{\text {er Ciclo }}$ & 15,8 & 36,8 & 28,9 & 18,4 & 0,0 \\
\hline Segundo Grado. $2^{\circ}$ Ciclo & 34,2 & 30,4 & 25,4 & 5,9 & 4,1 \\
\hline Tercer Grado. $7^{\text {per }}$ Ciclo & 24 & 45,1 & 22,1 & 7,4 & 0,8 \\
\hline Tercer Grado. $2^{\circ}$ Ciclo & 27,6 & 43,5 & 21,6 & 6,0 & 1,3 \\
\hline \multirow[t]{2}{*}{ Tercer Grado. $3^{\text {er Ciclo }}$} & 28,0 & 39,8 & 24,7 & 3,2 & 4,3 \\
\hline & \multicolumn{5}{|c|}{$\chi^{2}(24, N=1.056)=65,216, p<0,05$} \\
\hline
\end{tabular}


Esta evidencia se refuerza a través de otras combinaciones significativas, en especial las que vinculan carecer de estudios y no verificar nunca, y disponer de una licenciatura 0 un grado y contrastar la información de manera habitual.

Algo muy similar ocurre con el hábitat de los entrevistados (tabla 7). Los datos ponen de manifiesto que los jóvenes que residen en municipios de gran tamaño acuden con mayor asiduidad a fuentes alternativas ante posibles fake news.

\section{TABLA 7}

Frecuencia de contraste de la información en función del tamaño del hábitat de los encuestados

\begin{tabular}{|c|c|c|c|c|c|}
\hline & $\begin{array}{c}\text { Siempre } \\
(\%)\end{array}$ & $\begin{array}{c}\text { Habitualm. } \\
(\%)\end{array}$ & $\begin{array}{c}\text { A veces } \\
(\%)\end{array}$ & $\begin{array}{c}\text { Rara vez } \\
(\%)\end{array}$ & $\begin{array}{c}\text { Nunca } \\
(\%)\end{array}$ \\
\hline $5.001-10.000$ hab, & 30,3 & 31,6 & 21,1 & 3,9 & 13,2 \\
\hline $10.001-20.000$ hab, & 23,5 & 26,5 & 37,8 & 12,2 & 0,0 \\
\hline $20.001-50.000$ hab, & 30,2 & 29,1 & 25,9 & 7,4 & 7,4 \\
\hline $50.001-100.000$ hab, & 26,4 & 38,8 & 27,1 & 3,9 & 3,9 \\
\hline Más de 100.000 hab, & 31,9 & 39,9 & 21,2 & 6,0 & 1,1 \\
\hline & & $\chi^{2}(16, N=1.059)=72,832,0<0,05$ & \\
\hline
\end{tabular}

Y en sentido contrario, el análisis de residuos tipificados muestra una asociación significativa desde el punto de vista estadístico entre vivir en municipios de entre 5.001 y 10.000 habitantes y no contrastar nunca las informaciones.

\subsection{Fuentes empleadas para verificar las fake news}

Los jóvenes andaluces recurren sobre todo a webs especializadas para comprobar las noticias que les resultan poco creíbles (70,1\% de incidencias). También suelen acudir a medios de comunicación generalistas $(40,2 \%)$ a familiares o amigos $(25,2 \%)$ y, ya de forma menos habitual, a organizaciones y asociaciones $(11,7 \%)$, youtubers o influencers $(10,7 \%)$, empresas $(9,6 \%)$ y líderes políticos $(6,3 \%)^{6}$. 
El estadístico de contraste chi-cuadrado indica la existencia de asociaciones significativas entre la fuente a la que se recurre para verificar una posible fake news y el sexo, la edad y el nivel formativo de los individuos. Como refleja la tabla 8 , la asiduidad con la que se comprueba la información a través de familiares y amigos $(T E=0,73)$, empresas $(\mathrm{TE}=0,89)$ y organizaciones y asociaciones $(\mathrm{TE}=0,91)$ depende del sexo del encuestado, siendo las opciones primera y tercera las preferidas por el colectivo femenino, y la segunda algo más frecuente entre el masculino.

TABLA 8

Fuentes de contraste de la información en función del sexo de los encuestados ${ }^{*}$

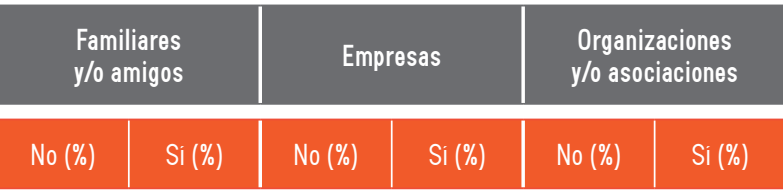

\begin{tabular}{|c|c|c|c|c|c|c|}
\hline Hombre & 77,9 & 22,1 & 87,8 & 12,2 & 91,2 & 8,8 \\
\hline \multirow[t]{2}{*}{ Mujer } & 71,5 & 28,5 & 93,1 & 6,9 & 85,3 & 14,7 \\
\hline & \multicolumn{2}{|c|}{$\begin{array}{c}\chi_{y}^{2}(1, N=1025)=5,089 \\
p<0,05\end{array}$} & \multicolumn{2}{|c|}{$\begin{array}{c}\chi_{y}^{2}(1, N=1025)=7,954 \\
p<0,05\end{array}$} & \multicolumn{2}{|c|}{$\begin{array}{c}\chi_{y}^{2}(1, N=1024)=7,944, \\
p<0,05\end{array}$} \\
\hline
\end{tabular}

* Solo se incluyen las categorías en las que el estadístico de contraste arroja resultados significativos.

Por otro lado, la edad parece incidir en que se contrasten las noticias por medio de páginas especializadas $(T E=0,105)$, youtubers e influencers $(T E=0,085) \mathrm{u}$ organizaciones y asociaciones $(\mathrm{TE}=0,102)$, como se recoge en la tabla 9. Así, los jóvenes de entre 15 y 20 años se inclinarían por YouTube, mientras que los mayores (de 20 a 24) preferirían las webs temáticas y las organizaciones. 
TABLA 9

Fuentes de contraste de la información en función de la edad de los encuestados*

\begin{tabular}{|c|c|c|c|c|c|c|}
\hline & \multicolumn{2}{|c|}{ Webs especializadas } & \multicolumn{2}{|c|}{ Youtubers/ influencers } & \multicolumn{2}{|c|}{$\begin{array}{l}\text { Organizaciones } \\
\text { y asociaciones }\end{array}$} \\
\hline & No (\%) & $\mathrm{Si}(\%)$ & № (\%) & $\mathrm{Si}(\%)$ & No (\%) & $\mathrm{Si}(\%)$ \\
\hline $15-20$ & 33,8 & 66,2 & 87,1 & 12,9 & 91,0 & 9,0 \\
\hline $20-24$ & 23,9 & 76,1 & 92,6 & 7,4 & 84,3 & 15,7 \\
\hline & \multicolumn{2}{|c|}{$\begin{array}{c}\chi_{y}^{2}(1, N=1024)=10,913 \\
p<0,05\end{array}$} & \multicolumn{2}{|c|}{$\begin{array}{c}\chi_{y}^{2}(1, N=1025)=6,937, \\
p<0,05\end{array}$} & \multicolumn{2}{|c|}{$\begin{array}{c}\chi_{y}^{2}(1, N=1025)=9,933, \\
p<0,05\end{array}$} \\
\hline
\end{tabular}

* Solo se incluyen las categorías en las que el estadístico de contraste arroja resultados significativos.

Para terminar, también el nivel educativo condiciona en esta variable. Su influencia es notoria en aquellos que optan por páginas especializadas $(T E=0,199)$, líderes políticos 0 partidos $(T E=0,191)$, youtubers o influencers $(T E=0,158)$, asociaciones $u$ organizaciones $(T E=0,120)$ y familiares o amigos ( $T E=0,117)$. Así, los encuestados que poseen menor formación suelen acudir a youtubers para corroborar las informaciones, y en segunda instancia, a su entorno más cercano, mientras que aquellos que disponen de un título superior prefieren visitar webs temáticas. En el resto de categorías mencionadas también se detectan diferencias por grupos, aunque sin alcanzar en ningún caso rangos significativos.

\section{Conclusiones y discusión}

Al plantear nuestro estudio, consideramos que trascender las muestras de conveniencia habituales en otros trabajos y analizar una franja etaria amplia (15-24 años), permitiría obtener un retrato más certero del impacto de las fake news entre los jóvenes 
-en concreto, los andaluces-, abarcando circunstancias sociodemográficas diversas. Además, podríamos obtener constataciones de mayor interés en relación con los objetivos planteados al comienzo de la investigación.

Con respecto a la frecuencia con que los jóvenes andaluces reciben fake news a través de las redes (01), parece que esta resulta extremadamente elevada. Los contenidos fraudulentos alcanzan a casi la totalidad de la muestra del estudio $(94,1 \%)$ al menos una vez al mes, y a más de la mitad varias veces por semana. Nos encontramos, por tanto, ante un fenómeno generalizado.

Cierto que el impacto difiere en función de algunas variables independientes: así, los datos revelan que, a mayor nivel de estudios, más habitual es recibir noticias falsas. Quizás esto se deba a que quienes no disponen de las herramientas cognitivas adecuadas por carecer del suficiente bagaje formativo, rara vez cuestionan la veracidad de los mensajes masivos, de modo que no son conscientes de estar siendo desinformados.

En segundo lugar, pretendíamos conocer las temáticas más habituales de las fake news que reciben los jóvenes, así como su procedencia (02). Parece haber dos ámbitos sobre los que pivotan este tipo de contenidos: sociedad -o mejor dicho, información sobre personajes de la vida pública-y política, por este orden. Ello contradice en parte a Vosoughi et al. (2018), para quienes el tema más común de las noticias falsas que circulan por las redes, a considerable distancia del resto de asuntos, es la política; pero parece que, entre los jóvenes -o al menos, entre los andaluces- predominan otro tipo de contenidos más frívolos. También Catalina-García et al. (2019) y Mendiguren et al. (2020) sostienen que las fake news más numerosas son las relacionadas con la política, pero quizá ello se deba al sesgo educativo de sus respectivas muestras, compuestas exclusivamente por estudiantes universitarios; en nuestro caso, el nivel formativo de los encuestados era más heterogéneo.

Aquí, el análisis de las variables independientes ha revelado cierto sesgo en función del sexo de los jóvenes: hay ámbitos temáticos con mayor impacto entre el colectivo femenino -sociedad y, en menor medida, cultura-, y otros cuya incidencia es más elevada entre el masculino -en especial, política, y de forma más discreta, deportes-.

Los medios de comunicación son identificados como fuente de noticias falsas por un sector importante de la juventud andaluza, lo cual se debe probablemente a la erosión de la credibilidad de las cabeceras y cadenas convencionales. Junto a ellos, la clase política, los youtubers y el entorno más cercano resultan también emisores habituales de fake news. De nuevo detectamos aquí diferencias según el nivel educativo de los entrevistados, ya que son los menos formados quienes en mayor medida identifican 
a influencers, familiares y amigos -las entidades menos solventes desde el punto de vista profesional- como fuente de informaciones falsas.

Finalmente, nos propusimos caracterizar el comportamiento de los jóvenes ante los contenidos fraudulentos (03). Al igual que constataban Catalina-Garcíaet al. (2017, 2019), en este segmento de la población la verificación de noticias poco fiables parece casi tan generalizada como su recepción, ya que alcanza al 90\% de la muestra, y se da sobre todo entre quienes tienen mayor nivel formativo y habitan en municipios muy poblados, algo que parece lógico.

Las webs temáticas y los medios de comunicación generalistas son las entidades a las que se acude con mayor frecuencia para verificar las informaciones. De nuevo hay aquí divergencias dependiendo de la edad y el nivel educativo: así, los jóvenes situados en la primera franja analizada (15-20 años) y con menos estudios se decantan por YouTube como fuente de contraste, mientras que los del segundo tramo (21-24), más formados, consultan de modo preferente las páginas especializadas.

En suma, nos encontraríamos ante un colectivo, el de los jóvenes andaluces, intensamente expuesto a las fake news, aunque con diferencias a veces significativas en función del sexo, la edad, el nivel formativo y el hábitat de los individuos. Queda para una futura investigación comprobar si las mismas pautas se repiten a escala nacional.

Lo que resulta imposible obviar ya es que las fake news se han instalado en el centro de los consumos informativos del segmento más joven de la audiencia. Ello obliga a plantear mecanismos de prevención y desactivación que pasan por potenciar -según la práctica totalidad de profesionales y estudiosos que se han ocupado del tema- la alfabetización mediática de la población. De lo contrario, los bulos masivos seguirán distorsionando el debate público indefinidamente, con las nefastas consecuencias que esto puede acarrear.

* Este estudio ha sido financiado con fondos del Plan Propio de Investigación de la Universidad de Málaga, a través de su programa de Proyectos Puente (convocatoria 2019).

\section{Bibliografía}

Alandete, D. (2019). Fake news: la nueva arma de destrucción masiva. Barcelona: Deusto. 
Allcott, H. \& Gentzkow, M. (2017). Social media and fake news in the 2016 election. Journal of Economic Perspectives, 31(2), 211-236. https://doi.org/10.1257/ jep.31.2.211

Amorós, M. (2018). Fake news: La verdad de las noticias falsas. Barcelona: Plataforma Actual.

Antunes, F. \& Nina, N. (2020). Información falsa en la red: Perspectiva de un grupo de estudiantes universitarios de comunicación en Portugal. Prisma Social, 29, 172-194. https:// revistaprismasocial.es/article/view/3593

Baider, F. H. \& Constantinou, M. (2017). Penelopegate: Fake news and online fake work over the 2017 French presidential campaign. Studia Romanica Posnaniensia, 44(3), 9-27.

Bakir, V. \& McStay, A. (2018). Fake news and the economy of emotions: Problems, causes, solutions. Digital Journalism, 6(2), 154-175.https://doi.org/10.1080/21 670811.2017 .1345645

Bakshy, E., Messing, S. \& Adamic, L. (2015). Exposure to ideologically diverse news and opinion on Facebook. Science, 348(6.239), 1.130-1.132.https://doi. org/10.1126/science.aaa1160

Bastos, M. T. \& Mercea, D. (2017). The Brexit botnet and user-generated hyperpartisan news. Social Science Computer Review, 37(1), 38-54. https://doi. org/10.1177/0894439317734157

Bovet, A. \& Makse, H. (2019). Influence of fake news in Twitter during the 2016 US presidential election. Nature Communications, 10(7). Disponible en: https://cutt. ly/ryiBxnH.Fecha de consulta:21-02-2020.

Brennen, J., Simon, F., Howard, P. \& Nielsen, R. (2020). Types, source, and claims of COVID-19 misinformation. Reuters Institute. Disponible en: https://cutt.ly/ Nt7wNEX. Fecha de consulta:10-04-2020.

Carrión, P. (2017). Trust in news. Kantar España Insights. Disponible en: https:// bit.ly/2I5XfJj. Fecha de consulta:01-03-2020. 
Catalina-García, B., Sousa, J. P. \& Cristina-Silva, L. C. (2019). Consumo de noticias y percepción de fake news entre estudiantes de Comunicación de Brasil, España y Portugal. Revista de Comunicación, 18(2), 93-115. https://doi.org/10.26441/ RC18.2-2019-A5

Catalina-García, B., Vozmediano, M. M. \& García-Jiménez, A. (2017). Los jóvenes universitarios y sus pautas de consumo y difusión de noticias según la tendencia ideológica. Fonseca, Journal of Communication, 15, 57-73. http://dx.doi. org/10.14201/fjc2017155773

Center for Internet Studies and Digital Life (2019). Digital News Report.es. Disponible en: https://www.digitalnewsreport.es/category/2019/. Fecha de consulta:10-03-2020.

Chadwick, A. (2013). The hybrid media system: Politics and power. Oxford: Oxford University Press.

Comisión Europea (2019). Eurobarómetro Standard 90. Opinión pública en la Unión Europea. Informe nacional. Disponible en: https://cutt.ly/gyiMRaB.Fecha de consulta:23-03-2020.

Consejo Audiovisual de Andalucía (2019). Barómetro Audiovisual de Andalucía 2019. Sevilla: Consejo Audiovisual de Andalucía, Junta de Andalucía. https://cutt. ly/qyhksOW

Fernández García, N. (2017). Fake news: Una oportunidad para la alfabetización mediática. Nueva Sociedad, 269, 66-77. https://cutt.ly/LyhkdDB

García, F., Gértrudix, M. \& Gértrudix, F. (2014). Análisis de la incidencia de la dieta de servicios digitales en la utilidad y confianza de la información en internet de los jóvenes universitarios. Communication \& Society, 27(1), 59-81.

García Santamaría, J. V. (2014). El negocio de la prensa digital. La Rioja: UNIR.

Gelfert, A. (2018). Fake news: a definition. Informal Logic, 38, 84-117. https:// doi.org/10.22329/il.v38i1.5068 


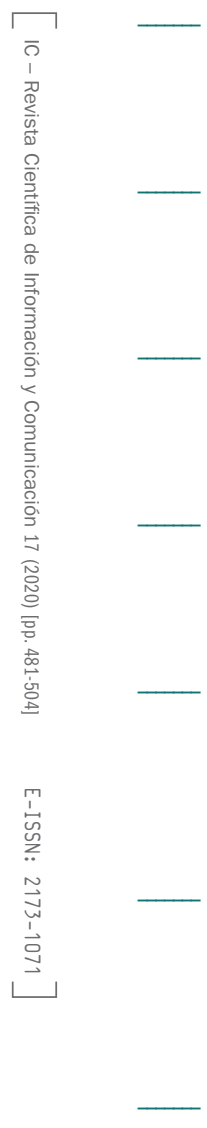

Grice, A. (2017). Fake news handed Brexiteers the referendum -and now they have no idea what they're doing. Independent (18 de febrero de 2017). Disponible en: https://ind.pn/20JoVpU. Fecha de consulta:21-02-2020.

Gottfried, J. \& Shearer, E. (2017). News use across social media platforms. Pew Research Center. Disponible en: https://pewrsr.ch/2A2orUT. Fecha de consulta:01-03-2020.

Griffin, A. (2016). What is "pizzagate"? The Hillary Clinton conspiracy theory that led to a man opening fire in a restaurant. Independent (05-12-2016). Disponible en: https://ind.pn/2xjmyWw. Fecha de consulta: 16-02-2020.

Guallar, J., Suau, J., Ruiz-Caballero, C., Suárez, A. \& Masip, P. (2016). Redistribución de noticias y debate público en las redes sociales. El Profesional de la Información, 25(3), 358-366. https:// cutt.ly/vyhkc1j.

Hernández-Conde, M. \& Fernández-García, M. (2019). Partidos emergentes de la ultraderecha: ¿fake news, fake outsiders? Vox y la web Caso Aislado en las elecciones andaluzas de 2018. Teknokultura. Revista de Cultura Digital y Movimientos Sociales, 16(1), 33-53. https://doi.org/10.5209/TEKN.63113

Herrero-Diz, P., Conde-Jiménez, J., Tapia-Frade, A. \& Varona-Aramburu, D. (2019). The credibility of online news: an evaluation of the information by university students. Cultura y Educación, 31(2), 407-435. https://doi.org/10.1080/1135 6405.2019.1601937

INE (2018). Demografía y población. Disponible en: https://cutt.ly/myi1sWu. Consultado el 13 de abril de 2019.

Madden, M., Lenhart, A. \& Fontaine, C. (2017). How youth navigate the news landscape. Miami: Knight Foundation.

Magallón, R. (2019a). Unfaking news. Madrid: Pirámide.

---- (2019b). Desinformación en campaña electoral. Telos. Disponible en:https:// cutt.ly/byi1M5v. Fecha de consulta:10-04-2020. 
Masip, P. et al. (2020). El consumo de información durante el confinamiento por el coronavirus: medios, desinformación y memes. Digilab. Disponible en: https:// cutt.ly/jyi0w0q.Fecha de consulta:21-04-2020.

Mendiguren, T., Pérez, J. \& Meso, K. (2020). Actitudes ante las fake news: Estudio del caso de los estudiantes de la Universidad del País Vasco. Revista de Comunicación, 19(1), 171-184. https://doi.org/10.26441/RC19.1-2020-A10

Mitchelstein, E. \& Boczkowski, P. (2018). Juventud, estatus y conexiones. Explicación del consumo incidental de noticias en redes sociales. Revista Mexicana de Opinión Pública, 24, 131-145. http://dx.doi.org/10.22201/ fcpys.24484911e.2018.24.61647

Newman, N., Dutton, W.H.\&Blank, G. (2012). Social media in the changing ecology of news: The fourth and fifth estate in Britain. International Journal of InternetScience, 7(1).Disponible en: https:// bit.ly/1FmAafU.Fecha de consulta:10-03-2020.

Nielsen, R. K.\&Schrøder, K. C. (2014). The relative importance of social media for accessing, finding, and engaging with news. Digital Journalism, 2(4), 472-489. https://doi.org/10.1080/21670811.2013.872420

Oliveira, J. \& Rossi, M. (2018). WhatsApp, el elemento distorsionador de la campaña en Brasil. El País (07-10-2018). Disponible en:https://bit.ly/2QxPleA. Fecha de consulta: 13-03-2020.

Palmer, E. (2017). The real problem with fake news. Educational Leadership, 75(3). Disponible en: https://cutt.ly/2yi5sxA. Fecha de consulta: 21-03-2020.

Parra, P. \& Oliveira, L. (2018). Fake news: Una revisión sistemática de la literatura. Observatorio (OBS), special issue, 54-78. https://doi.org/10.15847/ obsOBS12520181374

Pauner-Chulvi, C. (2018). Fake news and freedom of expression and information. The control of information contents on the network. Teoría y Realidad Constitucional, 41, 297-318. https://doi.org/10.5944/trc.41.2018.22123 


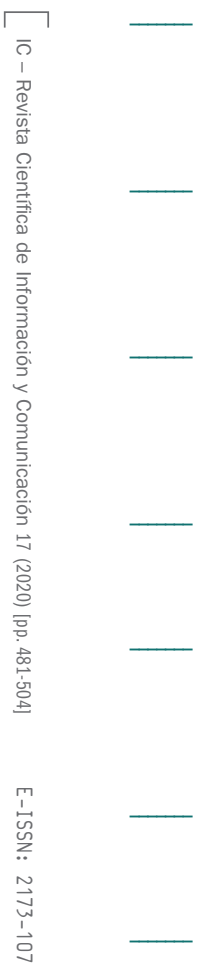

Salas, J. (2018). La información falsa llega más lejos, más rápido y a más gente que la verdadera. El País (08-03-2018). Disponible en: https://bit.ly/2lccNuE. Fecha de consulta: 12-03-2020.

Shao, C., Ciampaglia, G., Varol, 0., Flammini, A. \& Menczer, F. (2017). The spread of fake news by social bots. arXiv, 1707.07592v3. Disponible en: https://arxiv.org/ abs/1707.07592.Fecha de consulta: 02-03-2020.

Silverman, C. (2016). This analysis shows how fake election news stories outperformed real news on Facebook. BuzzFeed News. Disponible en: https://bzfd. it/2 puFLNW.Fecha de consulta: 27-02-2020.

Subramanian, S. (2017). Inside the Macedonian fake-news complex. Wired. Disponible en: https:// bit.ly/2kphWY3. Fecha de consulta: 27-02-2020.

Tandoc, E. C., Jenkins, J. \& Craft, S. (2019). Fake news as a critical incident in journalism. Journalism Practice, 13(6), 673-689. https:// doi.org/10.1080/1751 2786.2018.1562958

Tandoc, E. C., Wei Lim, Z. \& Ling, R. (2018). Defining “Fake news”. Digital Journalism, 6(2), 137-153. https:// doi.org/10.1080/21670811.2017.1360143

Taylor, P. (2013). Munitions of the mind: A history of propaganda. Oxford: Oxford University Press. 